\title{
Validation of Indian Council of Medical Research Neurocognitive Tool Box in Diagnosis of Mild Cognitive Impairment in India: Lessons from a Harmonization Process in a Linguistically Diverse Society
}

\author{
Ramshekhar N. Menon ${ }^{a} \quad$ Feba Varghese $^{b}$ Avanthi Paplikar ${ }^{b}$ Shailaja Mekalac,d Suvarna Alladib,d \\ Meenakshi Sharma ${ }^{e}$ Saroja Aralikatte Onkarappa ${ }^{f}$ Divyaraj Gollahallid Aparna Dutt ${ }^{g, h}$ \\ Amitabha Ghosh ${ }^{9}$ Rupinder Singh Dhaliwal ${ }^{e}$ Roopa Hooda' Gowri K. Iyer ${ }^{\text {d,j }}$ \\ Sunitha Justus $^{d}$ Rajmohan Kandukuri ${ }^{d}$ Subhash Kaul ${ }^{d, k}$ Arfa Banu Khan ${ }^{f}$ Ranita Nandig \\ Jwala Narayanan' Ashima Nehrai Padma M. Vasanthai Apoorva Pauranik ${ }^{\mathrm{m}}$ Robert Mathew ${ }^{\mathrm{n}}$ \\ Subasree Ramakrishnan ${ }^{b}$ Lekha Sarath $^{a}$ Urvashi Shah $^{\circ}$ Manjari Tripathi ${ }^{i}$ \\ Sylaja Padmavathy Narayana ${ }^{\mathrm{a}}$ Ravi Prasad Varma ${ }^{\mathrm{a}} \quad$ Mansi Verma $^{\mathrm{i}} \quad$ Yeshaswini Vishwanath $^{\mathrm{I}}$ \\ aDepartment of Neurology, Sree Chitra Tirunal Institute for Medical Sciences and Technology, \\ Thiruvananthapuram, India; ${ }^{b}$ Department of Neurology, National Institute of Mental Health and Neurosciences, \\ Bengaluru, India; 'Department of Psychiatry and Psychotherapy, University Hospital, LMU Munich, Munich, Germany; \\ 'Department of Neurology, Nizam's Institute of Medical Sciences, Hyderabad, India; 'Indian Council of Medical Research, \\ New Delhi, India; fJawaharlal Nehru Medical College, KLE Academy of Higher Education and Research, Belagavi, India; \\ ${ }^{9}$ Apollo Gleneagles Hospital, Cognitive Neurology Unit, Kolkata, India; h' Duttanagar Mental Health Centre, \\ Neuropsychology \& Clinical Psychology Unit, Duttanagar, Kolkata, India; 'Departments of Neurology and \\ Neuropsychology, All India Institute of Medical Sciences, Delhi, India; Indian Institute of Public Health, Hyderabad, India; \\ kDepartment of Neurology, Krishna Institute of Medical Sciences, Hyderabad, India; 'Manipal Hospitals, Bengaluru, India;

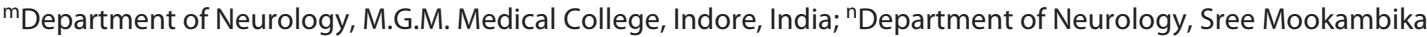 \\ Institute of Medical Sciences, Tamil Nadu, India; ${ }^{\circ}$ King Edward Memorial Hospital, Mumbai, India
}

\section{Keywords}

Neuropsychological test battery · Mild cognitive impairment · Harmonization · Validity · Vascular cognitive impairment

\section{Abstract \\ Background/Aims: In a linguistically diverse country such as India, challenges remain with regard to diagnosis of early cognitive decline among the elderly, with no prior attempts made to simultaneously validate a comprehensive battery of tests across domains in multiple languages. This study aimed to determine the utility of the Indian Council of Medical Re-}

search-Neurocognitive Tool Box (ICMR-NCTB) in the diagnosis of mild cognitive impairment $(\mathrm{MCl})$ and its vascular subtype (VaMCl) in 5 Indian languages. Methods: Literate subjects from 5 centers across the country were recruited using a uniform process, and all subjects were classified based on clinical evaluations and a gold standard test protocol into normal cognition, $\mathrm{MCl}$, and $\mathrm{VaMCl}$. Following adaptation and harmonization of the ICMR-NCTB across 5 different Indian languages into a composite $Z$ score, its test performance against standards, including sensitivity and specific-

Ramshekhar N. Menon, Suvarna Alladi, Amitabha Ghosh, and Manjari Tripathi are study site principal investigators.

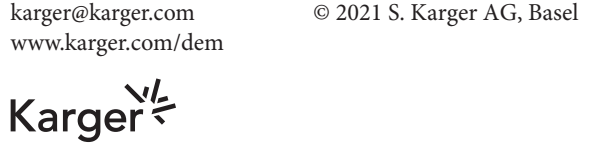


ity of the instrument as well as of its subcomponents in diagnosis of $\mathrm{MCl}$, was evaluated in age and education unmatched and matched groups. Results: Variability in sensitivity-specificity estimates was noted between languages when a total of 991 controls and 205 patients with $\mathrm{MCl}(157$ $\mathrm{MCl}$ and $48 \mathrm{VaMCl}$ ) were compared due to a significant impact of age, education, and language. Data from a total of 506 controls, 144 patients with $\mathrm{MCl}$, and 46 patients with $\mathrm{VaMCl}$ who were age- and education-matched were compared. Post hoc analysis after correction for multiple comparisons revealed better performance in controls relative to all-cause $\mathrm{MCl}$. An optimum composite Z-score of -0.541 achieved a sensitivity of $81.1 \%$ and a specificity of $88.8 \%$ for diagnosis of all-cause $\mathrm{MCl}$, with a high specificity for diagnosis of $\mathrm{VaMCl}$. Using combinations of multiple-domain 2 test subcomponents retained a sensitivity and specificity of $>80 \%$ for diagnosis of $\mathrm{MCl}$. Conclusions: The ICMR-NCTB is a "first of its kind" approach at harmonizing neuropsychological tests across 5 Indian languages for the diagnosis of $\mathrm{MCl}$ due to vascular and other etiologies. Utilizing multiple-domain subcomponents also retains the validity of this instrument, making it a valuable tool in $\mathrm{MCl}$ research in multilingual settings.

(C) 2021 S. Karger AG, Basel

\section{Introduction}

The high prevalence of Alzheimer's disease (AD) among the elderly has led to the identification of a detectable prodromal phase of $\mathrm{AD}$, an entity encompassed under the broad terminology "mild cognitive impairment" (MCI) [1-3]. Even when categorized into clinical subtypes, MCI continues to be a heterogeneous concept. While clinical, neuropsychological, and imaging biomarkers are used to diagnose MCI, variability in neuropsychological tests and diagnostic criteria employed across studies accounts for differences in diagnostic approaches and prediction of cognitive trajectory [4].

While most studies on amnestic MCI have focused on the entity as prodromal $\mathrm{AD}$, investigation into $\mathrm{MCI}$ as a forerunner of other possible dementias, such as vascular dementia, is rarely evident [5-8]. The paucity of literature stems from the fact that studies on MCI largely focus on the memory domain without assessing the executive or visuospatial domains which are crucial to detect vascular cognitive impairment (VCI) [9]. VCI forms the second largest group of major neurocognitive disorders in India and other developing countries $[10,11]$, and therefore, studies on MCI need to assess a more global profile of cognitive deficits to detect early vascular MCI (VaMCI) $[12,13]$.

Most studies in India have so far relied on MCI diagnosis using neuropsychological batteries developed/ adapted in only 1 language. However, in multicultural and multilingual societies unique to India, a nationally harmonized simultaneous validation process for diagnosis of MCI has not been attempted [14-16]. An important step toward addressing gaps in MCI diagnosis is to develop a common set of screening and diagnostic tools for free use in the context of linguistic and educational diversity in a vast country like India, which are standardized for both local and global use to help diagnose MCI and its subtypes. This should be done in a uniform manner in order to improve prognostication and initiate potential intervention strategies. It is with this intent that the Indian Council of Medical Research (ICMR) organized a task force with an expert group involving centers across the country to harmonize development of an ICMR-Neurocognitive Tool Box (NCTB) to diagnose dementia, MCI, and their subtypes [17]. The comprehensive test battery includes tests of attention/executive function, memory, language, and visuospatial functions, and questionnaires for functional activities, behavioral changes, and quality of life. Since the protocol was developed to enable diagnosis of dementia and MCI due to both degenerative disease and stroke, tests to detect focal cognitive deficits common in stroke, such as aphasia and spatial hemineglect, were specifically included. The primary aims of this sub-study on the ICMR-NCTB were to (a) ascertain its utility in the diagnosis of MCI and VaMCI in India by harmonizing cognitive assessment across 5 languages and (b) to attempt a unique validation of the instrument combining age- and education-matched groups using averaged composite scores derived from this harmonization process.

\section{Methods}

\section{Participants}

Research groups that participated in the development of the battery were in 5 academic institutions across India: Delhi, Kolkata, Hyderabad, Bengaluru, and Trivandrum. Language versions planned included Hindi, Bengali, Telugu, Kannada, and Malayalam, respectively. The detailed methodology, references for test selection and validation, as well as participating subject cohorts have been detailed previously in our study design paper [17]. Literate participants from each institution were selected and placed into 3 groups, that is, controls, MCI, and VaMCI following stroke as per current criteria based on their performance on gold standard 
cognitive tools (Addenbrooke's Cognitive Examination-III [ACEIII], Rey Auditory Verbal Learning Test [RAVLT], and Color Trails Test [CTT]) and a clinical interview conducted by a practicing clinician. Language-wise cutoffs as well as common cutoffs in age and education bands in ACE-III adaptations have been published recently [18]. Impairment on at least 2 out of the aforementioned 3 tests was mandatory for inclusion as MCI/VaMCI. The vernacular adaptations of the short form of the Informant Questionnaire on Cognitive Decline in the Elderly (short IQCODE) were also used in order to evaluate subjective cognitive impairment during the process of screening. A diagnosis of MCI and dementia was made based on the modified Petersen criteria for MCI and Diagnostic \& Statistical Manual of Mental Disorders (DSM-IVTR; American Psychiatric Association, 2000) criteria for dementia [19]. For MCI, patients should have reported subjective cognitive impairment with preserved general cognition. Objective evidence of impairment required that a subject scored $<1.5$ SD from the norm on at least 2 gold standard tests, as enumerated earlier. Inclusion criteria for the diagnosis of VaMCI following stroke were based on an antecedent history of documented stroke (diagnosed based on clinical symptoms and signs, with radiological evidence of infarct/s on CT scan or MRI) along with subjective cognitive disturbances following the stroke in addition to the MCI criteria as detailed below [20]:

1. Age $>40$ years.

2. A score of $\leq 5$ on the National Institute of Health Stroke Scale (NIHSS).

3. Subjective cognitive impairment following the index stroke episode with a score of $\leq 1$ on the Clinical Dementia Rating Scale (CDR).

4. Objective evidence of cognitive impairment on neuropsychological testing defined based on test score below mean $-1.5 \mathrm{SD}$ from the normative mean score for the test in a specific domain.

5. Absence of clinically significant anxiety/depression.

6. No prior history of subjective cognitive impairment before the stroke with a score of $\leq 2$ on the Modified Ranking Score (MRS). Subjects with isolated subclinical evidence of cerebrovascular disease on MRI or patients with subcortical ischemic VCI with no prior strokes were not included in the study to ensure a homogeneous VaMCI cohort could be recruited.

Inclusion criteria for controls are as follows:

1. Age $>40$ years.

2. No subjective cognitive disturbances on informant questionnaire.

3. No subjective complaints and scored normally on ACE-III, CDR, RAVLT, and CTT.

4. No evidence of head injury, infections, or neurological/systemic problems that can cause cognitive impairment.

5. No major systemic medical or psychiatric conditions that can interfere with cognition.

6. No significant hearing or visual impairment that can interfere with cognitive testing.

\section{Indian Council of Medical Research-Neurocognitive Tool Box}

ICMR-NCTB measures were selected from existing well-validated test batteries that have been extensively used in both highand low-income countries. If required, tests were adapted to the Indian context, as detailed previously [17]. The final battery comprised standardized tests covering 4 cognitive domains: (1) attention and executive functions using the Trail Making Test A\&B
(TMT A\&B) and category fluency for animals, (2) episodic memory using the Verbal Learning Test from Kolkata Cognitive Screening battery (delayed recall and total learning) and Modified Taylor Complex Figure (MTCF) test delayed recall, (3) language using the Picture Naming Test (PNT), and (4) visuospatial functions using the MTCF copy and delayed recall scores. This battery was validated in 5 Indian languages (Hindi, Bengali, Telugu, Kannada, and Malayalam). The total sample was also divided on the basis of years of education. In this study, only participants with at least 1 year of formal education and functional literacy, defined as "the ability to read and write in any language" (Indian Census 2011) were included. Controls were randomly drawn from attendants of the outpatients of the neurology, geriatric, and internal medicine clinics. Some participants were also recruited from senior citizen associations as well as community centers. The detailed medical history of the control participants was collected to determine their eligibility for participation in the study. The battery was administered by trained psychologists who were familiarized with the administration and interpretation of test batteries in a harmonization workshop, as detailed in our process paper [17]. The severity of cognitive impairment in patients was assessed using the CDR [21, 22]. Diagnoses of MCI/VaMCI and inclusion/exclusion criteria for controls were examined by the site principal investigator who is a neurologist experienced in diagnosis of cognitive disorders for $>5$ years. Basic demographic and social information was obtained by using a standard template.

\section{Statistical Analysis}

To compare demographic and clinical data of patients with MCI and controls, independent samples $t$ test for normally distributed continuous data or Mann-Whitney $U$ test for non-normal data, $\chi^{2}$ tests or Fisher's exact tests for categorical data, and trend test for ordinal data were used as appropriate. The ICMR-NCTB test scores were analyzed using univariate GLM to reveal the difference in the healthy controls and MCI patients. To determine the external validity of the battery, test scores were converted to standardized $\mathrm{Z}$ scores adjusting with age and education bands. The TMT time scores were multiplied by -1 after standardization. A composite score (combined score of TMT A\&B, category fluency, VLT-TL and DR, PNT, MTCF copy, and MTCF DR) was calculated from the averaged $\mathrm{Z}$ score. For the calculated composite scores, ROC analysis was done for overall MCI subjects (MCI + VaMCI) and separately for MCI and VaMCI subjects. AUC, sensitivity, and specificity were determined using ROC curve analysis for each language, followed by the composite battery averaging scores of age- and education-matched groups across languages. Optimum cutoff scores were established with corresponding sensitivity and specificity levels. All statistical analyses were performed using SPSS Statistics for Windows, version 23.0 (IBM Corporation, Armonk, NY, USA).

\section{Results}

\section{Demography}

A total of 1,196 participants were recruited, which included 991 controls and 205 patients with MCI (157 MCI and $48 \mathrm{VaMCI})$. Subsequently, after matching groups for
Five-Language Validation of a

Neurocognitive Toolbox in MCI
Dement Geriatr Cogn Disord 2020;49:355-364 DOI: $10.1159 / 000512393$ 
Table 1. Demographic characteristics of controls and patients with MCI

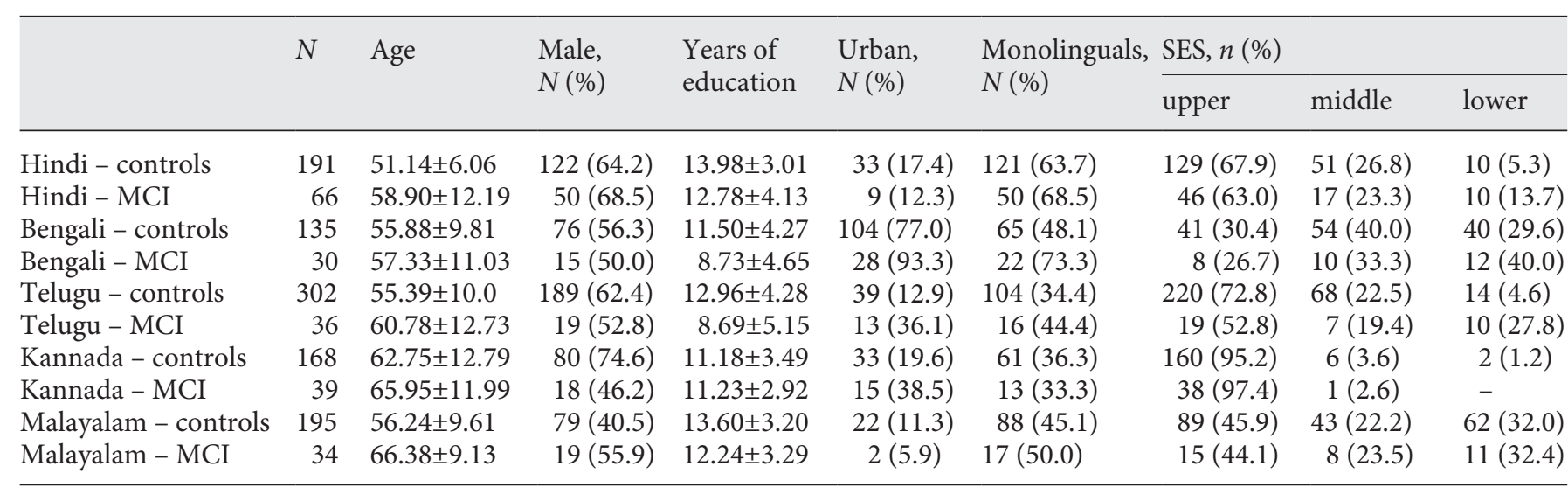

MCI, mild cognitive impairment.

Table 2. Demographic characteristics of age- and educationmatched patients with MCI and controls

\begin{tabular}{lccc}
\hline & $\begin{array}{l}\text { Controls } \\
(506)\end{array}$ & $\begin{array}{l}\text { MCI } \\
(190)\end{array}$ & $p$ value \\
\hline Age, years (mean, SD) & $62.7 \pm 8.8$ & $61.3 \pm 12.1$ & 0.158 \\
Education, years (mean, SD) & $12.0 \pm 3.6$ & $11.7 \pm 3.7$ & 0.298 \\
Male, $n$ (\%) & $287(56.7)$ & $112(58.9)$ & 0.597 \\
Urban, $n$ (\%) & $62(12.3)$ & $33(17.4)$ & 0.010 \\
Monolinguals, $n(\%)$ & $224(44.3)$ & $103(54.2)$ & 0.024 \\
\hline SES, $n(\%)$ & & & \\
Upper & $319(63.2)$ & $118(62.1)$ & 0.509 \\
Middle & $115(22.8)$ & $39(20.5)$ & \\
Lower & $71(14.1)$ & $33(17.4)$ & \\
\hline Language & & & \\
Hindi & 53 & 62 & $<0.001$ \\
Bengali & 77 & 28 & \\
Telugu & 149 & 28 & \\
Kannada & 111 & 39 & \\
Malayalam & 116 & 33 & \\
\hline
\end{tabular}

MCI, mild cognitive impairment.

age and education, 506 controls, 144 patients with MCI, and 46 patients with VaMCI were included in the next level of analysis. Demographic characteristics in each group are summarized in Tables 1 and 2 . Table 1 gives the distribution of the entire cohort with center-wise language-specific distributions, and Table 2 gives the ageand education-matched distributions combining the data from all 5 centers.

\section{Gold Standard Tests}

As the diagnosis of MCI was based on the gold standard tests, differences in test are evident from Table 3 for the entire data set within languages and Table 4 for age- and education-matched groups. Color Trails Test-A (CTT-A) in Telugu and RAVLT delayed recall (RAVLT-DR) in Kannada showed no differences in MCI-control comparison. As shown in Table 4, in the combined age- and educationmatched analysis, the MCI cohort demonstrated significant impairment on ACE-III and RAVLT-DR compared to controls, with no differences in CTT-A\&B and IQCODE. Although clinically significant anxiety and depression were excluded, Hospital Anxiety and Depression Scale (HADS) scores were significantly higher among MCI. The post hoc analysis of Tukey test revealed that the mean score for ACEIII was statistically significant between controls-MCI, controls-VaMCI, and VaMCI-MCI $(p<0.001)$.

\section{Utility of ICMR-NCTB for Diagnosis of MCI}

Analysis of performance across groups, as shown in Tables 5 and 6 , revealed that controls performed significantly better than patients with MCI on all the cognitive tests. The ICMR-NCTB components of domain-specific tests for attention-executive functions, episodic memory, language, and visuospatial functions demonstrated significant differences between MCI and controls in all languages, with the exception of category fluency in Hindi and Bengali as well as verbal list learning and visual memory scores in Kannada. The sensitivity and specificity estimates using ROC curves for diagnosis of all-cause MCI, vascular MCI, and non-vascular $\mathrm{MCI}$ in individual languages and for the combined averaged $\mathrm{Z}$ score estimates are depicted in Ta- 


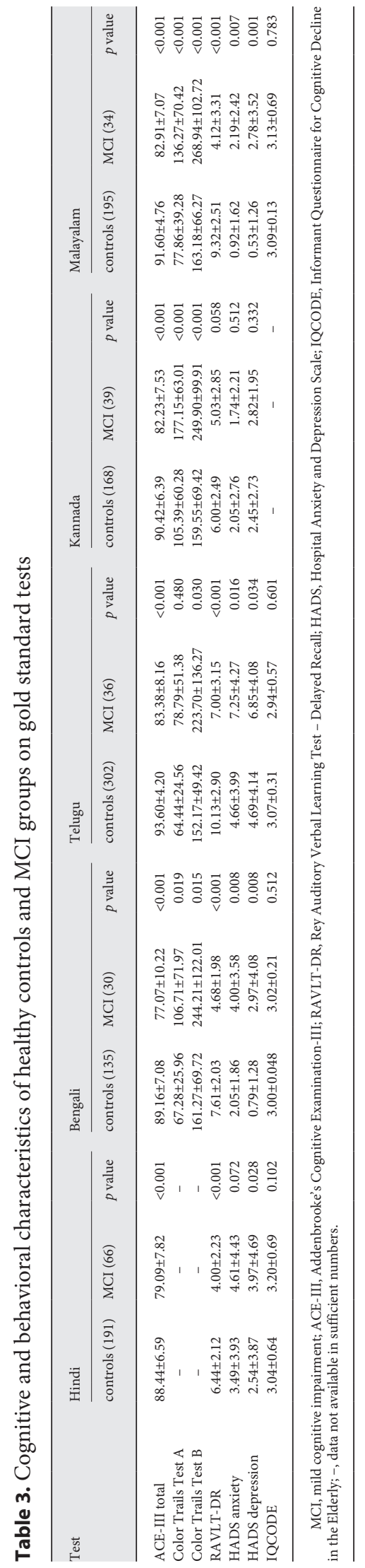

Five-Language Validation of a Neurocognitive Toolbox in MCI
Table 4. Cognitive and behavioral characteristics of age- and education-matched healthy controls and MCI groups on gold standard tests

\begin{tabular}{lccr}
\hline & Controls (506) & MCI (190) & $p$ value \\
\hline ACE-III total & $90.6 \pm 5.9$ & $80.8 \pm 8.3$ & $<0.001$ \\
Color Trails Test A & $81.1 \pm 37.1$ & $87.4 \pm 86.1$ & 0.220 \\
Color Trails Test B & $167.3 \pm 64.3$ & $165.6 \pm 149.5$ & 0.850 \\
RAVLT-DR & $7.7 \pm 2.8$ & $4.8 \pm 2.9$ & $<0.001$ \\
HADS anxiety & $2.4 \pm 3.1$ & $3.7 \pm 3.9$ & $<0.001$ \\
HADS depression & $2.2 \pm 3.2$ & $3.6 \pm 3.9$ & $<0.001$ \\
IQCODE & $3.0 \pm 0.4$ & $3.1 \pm 0.6$ & 0.128 \\
\hline
\end{tabular}

MCI, mild cognitive impairment; ACE-III, Addenbrooke's Cognitive Examination-III; RAVLT-DR, Rey Auditory Verbal Learning Test - Delayed Recall; HADS, Hospital Anxiety and Depression Scale; IQCODE, Informant Questionnaire for Cognitive Decline in the Elderly.

bles 7 and 8 and Figures 1 and 2. The overall best estimates of sensitivity in individual language versions for diagnosis of MCI ranged between 96.4 and $69.2 \%$ and specificity between 89.4 and $79.6 \%$. GLM analysis on the data from unmatched healthy controls when compared between centers revealed that education and language have significant independent main effects on ICMR-NCTB composite score (age: $F(1,921)=3.09 ; p=0.079$; education: $F(1,921)=$ $110.79 ; p<0.001$; language: $F(4,921)=15.06 ; p=0.001)$. After comparison between matched healthy controls, it was found that age, education, and language had no significant independent main effect on ICMR-NCTB composite score (age: $F(1,502)=0.056 ; p=0.813$; education: $F(1,502)=0.235 ; p=0.628$; language: $F(4,502)=3.45 ; p=$ $0.061)$. Since, there was only a nonsignificant language effect on ICMR NCTB score, we attempted to validate the battery using the averaged composite $\mathrm{Z}$ scores among ageand education-matched groups. Best estimates of sensitivity and specificity in the entire cohort for diagnosis of allcause MCI were 81.1 and $88.8 \%$, respectively.

Further estimates of the instrument performance when using a combination of various subcomponents in each domain, namely attention-executive function (TMT $\mathrm{B} \& \mathrm{~W}$ and category fluency), memory-verbal/visual (VLT and MTCF-learning and DR scores), language (PNT), and visuospatial (MTCF copy and DR), are shown in online suppl. Table 1 and Figure 1 (for all online suppl. material, see www.karger.com/doi/10.1159/000512393). The composite scores demonstrated greater validity for diagnosis of MCI as opposed to various combinations of these domain-specific subcomponent scores. 


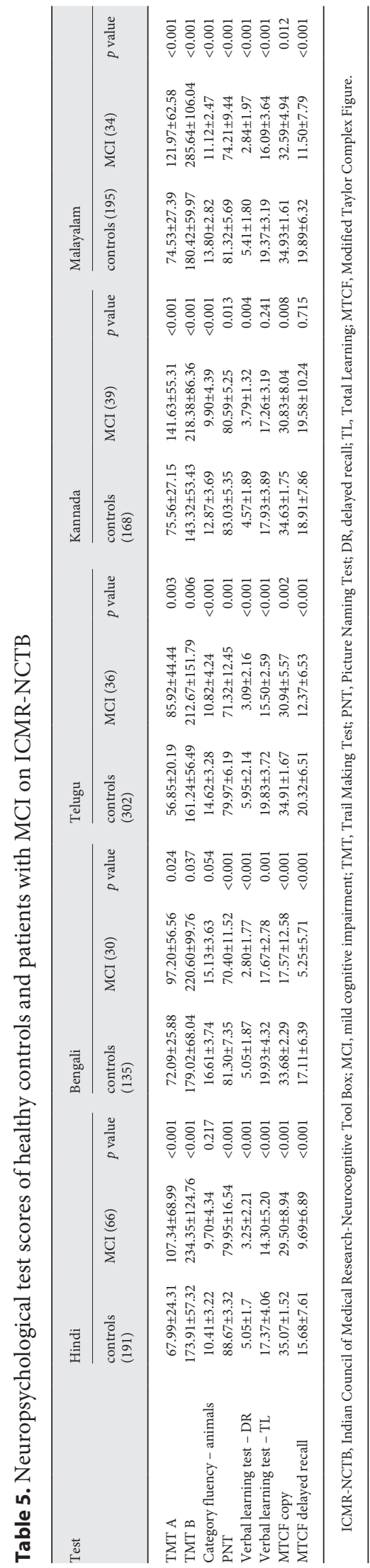

Table 6. Neuropsychological test scores across languages of ageand education-matched healthy controls and patients with MCI on ICMR-NCTB

\begin{tabular}{lccc}
\hline & $\begin{array}{l}\text { Controls } \\
(506)\end{array}$ & $\begin{array}{l}\text { MCI } \\
(190)\end{array}$ & $p$ value \\
\hline TMT A & $73.1 \pm 25.8$ & $116.9 \pm 59.6$ & $<0.001$ \\
TMT B & $177.2 \pm 60.3$ & $225.7 \pm 64.5$ & $<0.001$ \\
Category fluency - animals & $13.4 \pm 3.6$ & $10.9 \pm 4.4$ & $<0.001$ \\
PNT & $81.7 \pm 6.3$ & $76.7 \pm 18.7$ & $<0.001$ \\
Verbal learning test - DR & $5.0 \pm 1.8$ & $3.3 \pm 1.9$ & $<0.001$ \\
Verbal learning test - TL & $18.4 \pm 3.6$ & $16.0 \pm 3.8$ & $<0.001$ \\
MTCF copy & $34.7 \pm 1.8$ & $29.3 \pm 9.1$ & $<0.001$ \\
MTCF delayed recall & $30.1 \pm 8.2$ & $11.9 \pm 9.5$ & $<0.001$ \\
\hline
\end{tabular}

MCI, mild cognitive impairment; ICMR-NCTB, Indian Council of Medical Research-Neurocognitive Tool Box; TMT, Trail Making Test; PNT, Picture Naming Test; DR, delayed recall; TL, Total Learning; MTCF, Modified Taylor Complex Figure.

Table 7. Sensitivity and specificity of ICMR-NCTB in diagnosing all MCI across languages at optimum cutoff values across 5 languages ( $\mathrm{Z}$ score calculated after adjusting for age and education bands)

\begin{tabular}{lllll}
\hline Language & $\begin{array}{l}\text { Cutoff } \\
\text { value }\end{array}$ & $\begin{array}{l}\text { Sensitivity, } \\
\%\end{array}$ & $\begin{array}{l}\text { Specificity, } \\
\%\end{array}$ & $\begin{array}{l}\text { Area under } \\
\text { curve }\end{array}$ \\
\hline Telugu & -0.560 & 71.4 & 89.4 & \\
& -0.408 & 85.7 & 85.2 & 0.925 \\
& -0.226 & 96.4 & 75.0 & \\
\hline Hindi & -0.566 & 77.4 & 79.6 & \\
& -0.409 & 80.6 & 72.4 & 0.796 \\
& -0.226 & 85.5 & 57.2 & \\
\hline Malayalam & -0.557 & 63.6 & 86.8 & \\
& -0.399 & 66.7 & 82.6 & 0.858 \\
& -0.227 & 81.8 & 73.7 & \\
\hline Bengali & -0.564 & 85.7 & 80.2 & \\
& -0.395 & 89.3 & 73.0 & 0.954 \\
& -0.221 & 89.3 & 62.7 & \\
\hline Kannada & -0.564 & 51.3 & 87.1 & 0.742 \\
& -0.400 & 59.0 & 74.8 & \\
& -0.228 & 69.2 & 64.6 & \\
\hline
\end{tabular}

ICMR-NCTB, Indian Council of Medical Research-Neurocognitive Tool Box; MCI, mild cognitive impairment. 




Fig. 1. ROC curve for overall MCI: language-wise variations in sensitivity-specificity estimates in 5 Indian languages on age- and education-unmatched group comparisons. MCI, mild cognitive impairment.

Table 8. Validity/sensitivity and specificity of ICMR-NCTB in diagnosing MCI at optimum cutoff values using ROC curves

\begin{tabular}{lllll}
\hline Diagnosis & $\begin{array}{l}\text { Cutoff } \\
\text { value }\end{array}$ & $\begin{array}{l}\text { Sensitivity, } \\
\%\end{array}$ & $\begin{array}{l}\text { Specificity, } \\
\%\end{array}$ & $\begin{array}{l}\text { Area under } \\
\text { curve }\end{array}$ \\
\hline All MCI & -0.544 & 80.5 & 84.6 & \\
& -0.545 & 80.5 & 84.0 & 0.892 \\
& -0.541 & 81.1 & 88.8 & \\
\hline MCI & -0.559 & 78.5 & 79.5 & \\
& -0.549 & 79.2 & 78.8 & 0.854 \\
& -0.532 & 80.6 & 77.9 & \\
\hline VaMCI & -0.755 & 70.8 & 86.1 & \\
& -0.725 & 71.5 & 85.3 & 0.820 \\
& -0.721 & 72.9 & 84.8 & \\
\hline
\end{tabular}

ICMR-NCTB, Indian Council of Medical ResearchNeurocognitive Tool Box; MCI, mild cognitive impairment; VaMCI, vascular MCI.

Five-Language Validation of a Neurocognitive Toolbox in MCI

\section{Discussion}

This study confirmed the utility of ICMR-NCTB as an effective instrument for diagnosis of MCI, including its vascular subtype, in hospital-based settings. A key outcome of this study was the development and validation of a comprehensive neurocognitive test battery that can be used to characterize and quantify deficits in MCI in a heterogeneous population. This was accomplished by studying a large cohort that constituted subjects from different regions within India, grouped by their linguistic profile. The development of a common methodology to study MCI across different languages will reduce variability in diagnosis in hospitals and clinics across India. A significant effect of education and language was noted when unmatched $\mathrm{MCI}$ and control groups were compared, thereby leading to variability in sensitivity and specificity between individ- 


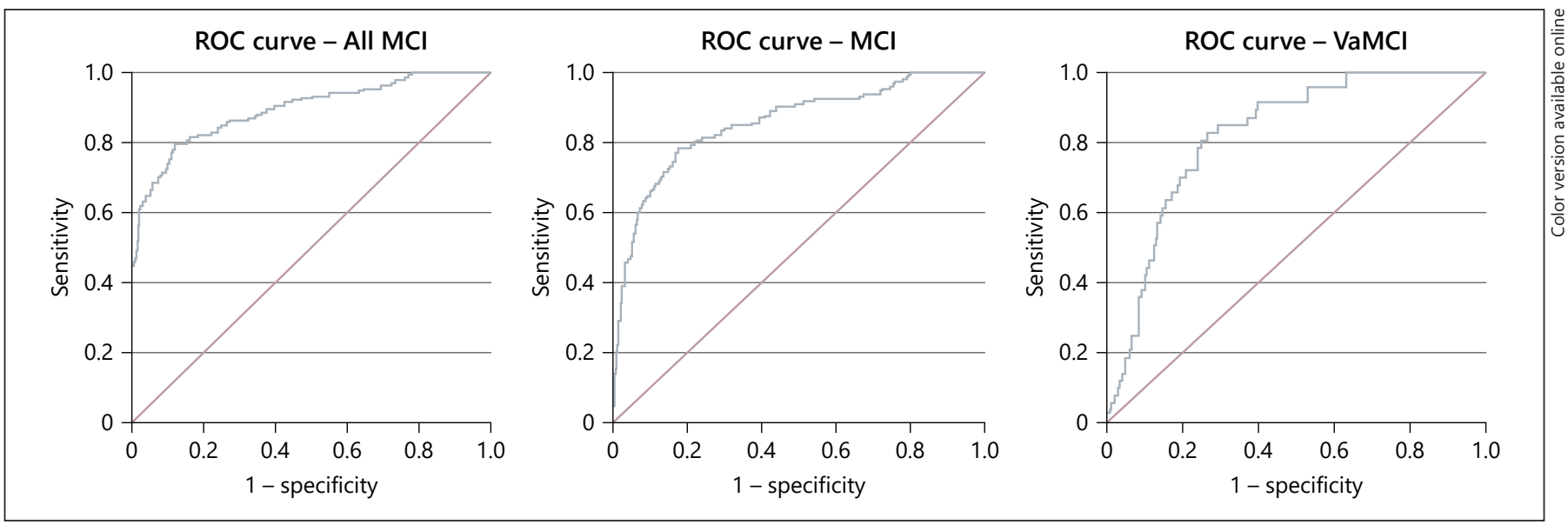

Fig. 2. ROC curve for overall MCI, MCI-other causes, and VaMCI among age- and education-matched groups across centers. MCI, mild cognitive impairment; VaMCI, vascular MCI.

ual language versions. The composite score generated after comparing age- and education-matched groups was significantly more sensitive and specific compared to combinations of domain-specific subcomponents. Thus, the ICMR-NCTB is demonstrably a valid instrument for use in both clinical and research settings across the country.

Critically analyzing the process of validation between languages indigenous to 5 regions across India, it is important to realize that Indian languages are diverse in their phonology, grammar, lexicon, and semantics [17]. This diversity is likely to have contributed to the variable sensitivity-specificity between languages. In an ideal world, the way to reconcile such language-related variations would be to test all language versions in the multilingual participants and investigate for differences in area under the curve on ROC [23]. However, it can be assumed that any differences due to the language-related variations would reflect equally at different levels of the $\mathrm{Z}$ score distributions and hence have minimal effect on the final averaged composite. This was also reiterated by the GLM results on the control data set in our study. This nonsignificant impact of language has also been similarly demonstrated in our recent 7-language validation study of ACE-III, which was used as one of the gold standard tests for this study [18]. We used composite $\mathrm{Z}$ scores based on the understanding that raw test scores, but not the standardized Z scores, would vary across languages/locations [24]. Cultural effects could have also played a role in differences in test performance across cohorts [25]. Therefore, any potential variability between centers or languages may be due to other unmeasured factors such as cultural/ethnic background and test administration methods but unlikely to be due to the 3 demographic factors: age, education, and language.

With a sensitivity of $81.1 \%$ and a specificity of $88.8 \%$, the utility of the ICMR-NCTB as a diagnostic instrument for MCI has been established. The debate regarding the diagnostic approach to MCI with consideration to the neuropsychological tests and the optimum number of tests to be used is yet unresolved [25-27]. In our study, the gold standard considered was clinical diagnosis associated with impairment on at least 2 out of 3 tests, which included a screening battery (ACE-III), word list learning and recall (RAVLT), and executive functioning (CTT A\&B). The absence of significant differences between the comparison groups on a pragmatic questionnaire-based screening instrument for dementia, that is, the IQCODE, further ensured that the clinical cohort was free of subjects who had progressed to dementia. While the feasibility and convenience of using a single cognitive screening instrument such as ACE-III and MOCA for the diagnosis of MCI cannot be undermined [28-32], the drawback is that impairment on a single domain is common in MCI but may not be sufficient to impact the total score. Therefore, we did not rely on a single global screening instrument alone for diagnosis.

In our study, utilizing the subcomponents of the ICMRNCTB, that is, using various combinations of memory, language, executive, and visuospatial functions tests, did not significantly improve the sensitivity and specificity for diagnosis of MCI. Although employing domain-specific measures alone may have a role in predicting conversion to dementia, their use in a heterogeneous entity such as $\mathrm{MCI}$ runs the risk of underdiagnosis. It is also recognized that the best validity for MCI diagnosis is seen when 2 tests in each domain are employed in composite. Recent evi- 
dence from the Framingham cohort demonstrated that only the 2 tests approach, that is, Jak/Bondi criteria (2 tests impaired/domain, $>1$ SD below normal) remained significantly associated with incident dementia on a combined statistical hazard model [33-35]. In the ICMR-NCTB as well, 2 measures of attention/executive functions and memory were included in order to increase its validity.

With a sensitivity of $72.9 \%$ and a specificity of $84.8 \%$ for diagnosis of VaMCI, it is evident that the ICMRNCTB is reasonably efficacious in the clinical and research setting for diagnosis of early cognitive impairment following stroke. An interesting finding was the higher HADS score in MCI compared to controls. Although clinically significant anxiety and depression were excluded, this finding was not unexpected, especially since depression is frequently associated with post-stroke cognitive impairment [36]. Given the high specificity of the NCTB, the composite scores could be useful as neuropsychological biomarkers of cognitive decline in stroke.

Despite being a harmonized multicentric and multilinguistic hospital-based study, certain limitations are evident. The ICMR-NCTB version employed is not suitable for illiterates, who form a significant proportion of the Indian population. An illiterate version of the test battery has been developed and will need to be evaluated for its validity among people with no/minimal education [17]. Sensitivity-specificity estimates could have been impacted by the small sample sizes in MCI and vascular-MCI in comparison to controls. The sensitivity and specificity of the battery will also depend on whether communitybased or hospital-based cohorts are being screened or diagnosed [29]. Reliability and stability of MCI diagnosis in the cohort will ideally require to be established in a longitudinal manner. A longitudinal follow-up with structural and metabolic imaging or CSF biomarkers will ascertain validity of domain-specific subscores [37] and etiology-specific MCI diagnosis using the ICMR-NCTB.

Our study thus establishes a benchmark for MCI research in India with well-defined norms with focused consideration of the unique linguistic and sociocultural diversity within the country. While a uniform pan-India measure of normal or impaired cognition across multicultural societies like India is not likely to be a reality, our exercise provides a platform to enable diagnostic comparisons of MCI across the entire country and also with populations around the world.

"Published in Celebration of the 30th Anniversary of the inception of Dementia and Geriatric Cognitive Disorders 1990-2020".

Five-Language Validation of a

Neurocognitive Toolbox in MCI

\section{Acknowledgements}

We would like thank the following individuals for their expertise extended toward the project and research design: (late) J.S. Chopra, (late) S.K. Das, S. Prabhakar, Narendra K. Arora, Anand Krishnan, M. Gourie-Devi (ICMR scientific experts), Prathiba Karanth, Sunil Kumar Ravi, P.S. Mathuranath, Ratnavalli Ellajosyula, and Annamma George. We would also like to thank the following people/organizations for providing us with permission to use and adapt the tests wherever required: John R. Hodges for ACE-III, Sang Yun Kim for Trail Making Test-B\&W, Anitha M. Hubley for MTCF, P.S. Mathuranath for PN test and IADL-E, Jeffery Cummings for NPI, and Anthony Jorm for IQCODE.

\section{Statement of Ethics}

This study was conducted with the Institution Ethics Committee approvals from AIIMS (Delhi), Nizam's Institute of Medical Sciences (Hyderabad), Apollo Gleneagles Hospital (Kolkata), NIMHANS (Bangalore), Jawaharlal Nehru Medical College (Belgavi), and SCTIMST (Trivandrum). Informed consent was taken from all the participants of this study.

\section{Conflict of Interest Statement}

The authors have no conflicts of interest to disclose

\section{Funding Sources}

This work was supported by Indian Council for Medical Research under Grant (SWG/Neuro/32/2017-NCD-1).

\section{Author Contributions}

R.N.M., S.A., M.S., A.G., and M.T. conceived and designed the research, applied for funding, coordinated the logistics, supervised data collection and analysis, and manuscript preparation and revision. F.V., A.P., S.M., and G.K.I. were involved in data collection, data organization and analysis, and manuscript preparation and revision. R.P.V. was involved in data analysis and critical revision. A.D., J.N., and A.N. were involved in neuropsychological test battery design, data analysis, and critical revision. D.G., R.H., S.J., R.K., A.B.K., R.N., S.R., L.S., M.V., and Y.V. were involved in data collection, organization, and analysis. R.S.D., S.A.O., S.K., P.M.V., A.P., R.M., U.S., and S.P.N. were involved in research design and critical revision of the final manuscript.

\section{Data Statement}

The data that support the findings of this study are available on request from the corresponding author. The data are not publicly available due to privacy and ethical restrictions. 


\section{References}

1 McKhann GM, Knopman DS, Chertkow H, Hyman BT, Jack CR Jr, Kawas CH, et al. The diagnosis of dementia due to Alzheimer's disease: recommendations from the National Institute on Aging-Alzheimer's Association workgroups on diagnostic guidelines for $\mathrm{Al}$ zheimer's disease. Alzheimers Dement. 2011 May;7(3):263-9.

2 Mathuranath PS, George A, Ranjith N, Justus S, Kumar MS, Menon R, et al. Incidence of Alzheimer's disease in India: a 10 years follow-up study. Neurol India. 2012 Nov-Dec;60(6):62530.

3 Viticchi G, Falsetti L, Buratti L, Sajeva G, Luzzi $\mathrm{S}$, Bartolini M, et al. Framingham risk score and the risk of progression from mild cognitive impairment to dementia. J Alzheimers Dis. 2017; 59(1):67-75.

4 Overton M, Pihlsgård M, Elmståhl S. Diagnostic stability of mild cognitive impairment, and predictors of reversion to normal cognitive functioning. Dement Geriatr Cogn Disord. 2019;48(5-6):317-29.

5 Elias MF, Beiser A, Wolf PA, Au R, White RF, D'Agostino RB. The preclinical phase of Alzheimer disease: a 22-year prospective study of the Framingham Cohort. Arch Neurol. 2000 Jun;57(6):808-13.

6 Petersen RC, Roberts RO, Knopman DS, Boeve BF, Geda YE, Ivnik RJ, et al. Mild cognitive impairment: ten years later. Arch Neurol. 2009 Dec;66(12):1447-55.

7 Ganguli M, Snitz BE, Saxton JA, Chang CC, Lee CW, Vander Bilt J, et al. Outcomes of mild cognitive impairment by definition: a population study. Arch Neurol. 2011 Jun;68(6):761-7.

8 Petersen RC. Clinical practice. Mild cognitive impairment. N Engl J Med. 2011 Jun 9;364(23): 2227-34.

9 Graham NL, Emery T, Hodges JR. Distinctive cognitive profiles in Alzheimer's disease and subcortical vascular dementia. J Neurol Neurosurg Psychiatry. 2004 Jan;75(1):61-71.

10 Alladi S, Kaul S, Meena AK, Somayajula S, Umadevi M, Reddy JM. Pattern of vascular dementia in India: study of clinical features, imaging, and vascular mechanisms from a hospital dementia registry. J Stroke Cerebrovasc Dis. 2006 Mar-Apr;15(2):49-56.

11 Moorhouse P, Rockwood K. Vascular cognitive impairment: current concepts and clinical developments. Lancet Neurol. 2008 Mar;7(3): 246-55.

12 Ihle-Hansen H, Thommessen B, Wyller TB, Engedal K, Øksengård AR, Stenset V, et al. Incidence and subtypes of MCI and dementia 1 year after first-ever stroke in patients without pre-existing cognitive impairment. Dement Geriatr Cogn Disord. 2011;32(6):401-7.

13 Divya KP, Menon RN, Varma RP, Sylaja PN, Thomas B, Kesavadas C, et al. Post-stroke cognitive impairment: a cross-sectional comparison study between mild cognitive impairment of vascular and non-vascular etiology. J Neurol Sci. 2017 Jan 15;372:356-62.
14 Das SK, Bose P, Biswas A, Dutt A, Banerjee TK, Hazra AM, et al. An epidemiologic study of mild cognitive impairment in Kolkata, India. Neurology. 2007 Jun 5;68(23):2019-26.

15 Menon R, Lekha V, Justus S, Sarma PS, Mathuranath P. A pilot study on utility of Malayalam version of Addenbrooke's Cognitive Examination in detection of amnestic mild cognitive impairment: a critical insight into utility of learning and recall measures. Ann Indian Acad Neurol. 2014 Oct;17(4):420-5.

16 Bharath S, Sadanand S, Kumar KJ, Balachandar $\mathrm{R}$, Joshi H, Varghese M. Clinical and neuropsychological profile of persons with mild cognitive impairment, a hospital based study from a lower and middle income country. Asian J Psychiatr. 2017 Dec;30:185-9.

17 Iyer GK, Paplikar A, Alladi S, Dutt A, Sharma M, Mekala S, et al. Standardising dementia diagnosis across linguistic and educational diversity: study design of the Indian Council of Medical Research-Neurocognitive Tool Box (ICMR-NCTB). J Int Neuropsychol Soc. 2020 Feb; 26(2):172-86.

18 Mekala S, Paplikar A, Mioshi E, Kaul S, Divyaraj G, Coughlan G, et al. Dementia diagnosis in seven languages: the Addenbrooke's Cognitive Examination-III in India. Arch Clin Neuropsychol. 2020 Jul 24;35(5):528-38.

19 Petersen RC. Mild cognitive impairment as a diagnostic entity. J Intern Med. 2004 Sep; 256(3):183-94.

20 Gorelick PB, Scuteri A, Black SE, Decarli C, Greenberg SM, Iadecola C, et al. Vascular contributions to cognitive impairment and dementia: a statement for healthcare professionals from the American Heart Association/American Stroke Association. Stroke. 2011 Sep;42(9): 2672-713.

21 Morris JC. The clinical dementia rating (CDR): current version and scoring rules. Neurology. 1993 Nov;43(11):2412-4

22 Woolf C, Slavin MJ, Draper B, Thomassen F, Kochan NA, Reppermund S, et al. Can the clinical dementia rating scale identify mild cognitive impairment and predict cognitive and functional decline? Dement Geriatr Cogn Disord. 2016;41(5-6):292-302.

23 Streiner DL, Cairney J. What's under the ROC? An introduction to receiver operating characteristics curves. Can J Psychiatry. 2007 Feb; 52(2):121-8.

24 Wright EJ, Grund B, Robertson K, Brew BJ, Roediger M, Bain MP, et al. Cardiovascular risk factors associated with lower baseline cognitive performance in HIV-positive persons. Neurology. 2010 Sep 7;75(10):864-73.

25 Manly JJ, Tang MX, Schupf N, Stern Y, Vonsattel JP, Mayeux R. Frequency and course of mild cognitive impairment in a multiethnic community. Ann Neurol. 2008 Apr;63(4):494-506.

26 Lopez OL, Jagust WJ, DeKosky ST, Becker JT, Fitzpatrick A, Dulberg C, et al. Prevalence and classification of mild cognitive impairment in the Cardiovascular Health Study Cognition
Study: part 1. Arch Neurol. 2003 Oct;60(10): 1385-9.

27 Schinka JA, Loewenstein DA, Raj A, Schoenberg MR, Banko JL, Potter H, et al. Defining mild cognitive impairment: impact of varying decision criteria on neuropsychological diagnostic frequencies and correlates. Am J Geriatr Psychiatry. 2010 Aug;18(8):684-91.

28 Nasreddine ZS, Phillips NA, Bédirian V, Charbonneau S, Whitehead V, Collin I, et al. The Montreal Cognitive Assessment, MoCA: a brief screening tool for mild cognitive impairment. J Am Geriatr Soc. 2005 Apr;53(4):695-9.

29 Larner AJ. Comparing diagnostic accuracy of cognitive screening instruments: a weighted comparison approach. Dement Geriatr Cogn Dis Extra. 2013 Jan;3(1):60-5.

30 Pendlebury ST, Mariz J, Bull L, Mehta Z, Rothwell PM. Impact of different operational definitions on mild cognitive impairment rate and MMSE and MoCA performance in transient ischaemic attack and stroke. Cerebrovasc Dis. 2013;36(5-6):355-62.

31 Williamson JC, Larner AJ. MACE for the diagnosis of dementia and MCI: 3-year Pragmatic Diagnostic Test Accuracy Study. Dement Geriatr Cogn Disord. 2018;45(5-6):300-7.

32 Pinto TCC, Machado L, Costa MLG, Santos MSP, Bulgacov TM, Rolim APP, et al. Accuracy and psychometric properties of the Brazilian version of the Montreal cognitive assessment as a brief screening tool for mild cognitive impairment and Alzheimer's disease in the initial stages in the elderly. Dement Geriatr Cogn Disord. 2019;47(4-6):366-74.

33 Jak AJ, Bondi MW, Delano-Wood L, Wierenga C, Corey-Bloom J, Salmon DP, et al. Quantification of five neuropsychological approaches to defining mild cognitive impairment. Am J Geriatr Psychiatry. 2009 May;17(5):368-75.

34 Jak AJ, Preis SR, Beiser AS, Seshadri S, Wolf PA, Bondi MW, et al. Neuropsychological criteria for mild cognitive impairment and dementia risk in the Framingham Heart Study. J Int Neuropsychol Soc. 2016 Oct;22(9):937-43.

35 Wong CG, Thomas KR, Edmonds EC, Weigand AJ, Bangen KJ, Eppig JS, et al. Neuropsychological criteria for mild cognitive impairment in the Framingham Heart Study's old-old. Dement Geriatr Cogn Disord. 2018;46(5-6):25365.

36 Terroni L, Sobreiro MFM, Conforto AB, Adda CC, Guajardo VD, de Lucia MCS, et al. Association among depression, cognitive impairment and executive dysfunction after stroke. Dement Neuropsychol. 2012 Jul-Sep;6(3): 152-7.

37 Belleville S, Fouquet C, Hudon C, Zomahoun HTV, Croteau J; Consortium for the Early Identification of Alzheimer's d-Q. Neuropsychological measures that predict progression from mild cognitive impairment to Alzheimer's type dementia in older adults: a systematic review and meta-analysis. Neuropsychol Rev. 2017 Dec;27(4):328-53. 